\title{
Evaluation of Cumulative and Conditional Antibiotic Release from Vancomycin-Embedded Fibrin Sealant and Its Antibacterial Activity : An In Vitro Study
}

\author{
Dong-Won Shin, Moon-Jun Sohn, ${ }^{1,2}$ Chong-Rae Cho, ${ }^{3}$ Hae-won Koo, ${ }^{1,2}$ Sang Won Yoon ${ }^{1,2,4}$ \\ Department of Neurological Surgery, Inje University Ilsan Paik Hospital, Inje University College of Medicine, Goyang, Korea \\ Neuroscience \& Radiosurgery Hybrid Research Center, Inje University Ilsan Paik Hospital, Inje University College of Medicine, Goyang, Korea \\ Department of Laboratory Medicine, ${ }^{3}$ Inje University Ilsan Paik Hospital, Inje University College of Medicine, Goyang, Korea \\ Department of Neurosurgery, Inje University Seoul Paik Hospital, Inje University College of Medicine, Seoul, Korea
}

Objective : Fibrin sealants have been used for hemostasis, sealant for cerebrospinal fluid leakage, and adhesive barrier in neurosurgery. Further, as its clinical use and role of an effective drug delivery vehicle have been proposed. This study was performed to measure antibacterial activity and continuous local antibiotic release from different concentrations of vancomycin-impregnated fibrin sealant in vitro.

Methods : Antibacterial activity was investigated by disk diffusion test by measuring the diameter of the growth inhibition zone of bacteria (methicillin-resistant Staphylococcus aureus, ATCC29213) from vancomycin-embedded fibrin sealant disc diluted at five different concentrations ( $\mathrm{C} 1-\mathrm{C} 5 ; 8.33,4.167,0.83,0.083$, and $0.0083 \mathrm{mg} / \mathrm{disc}$, respectively). Continuous and conditioned release of vancomycin concentration (for 2 weeks and for 5 days, respectively) were also measured using high-performance liquid chromatography (HPLC) method. To mimic the physiologic wound conditions with in vitro, conditioned vancomycin release in phosphate buffer solution (PBS) was measured and replaced PBS for five consecutive days, half a day or completely daily.

Results : In the disk diffusion test, the mean diameters of bacterial inhibition zone were $2.54 \pm 0.07 \mathrm{~cm}, 2.61 \pm 0.12 \mathrm{~cm}$, and $2.13 \pm 0.15$ $\mathrm{cm}(\mathrm{C} 1, \mathrm{C} 2$, and $\mathrm{C} 3$ respectively) but $1.67 \pm 0.06 \mathrm{~cm}$ and $1.23 \pm 0.15 \mathrm{~cm}$ in $\mathrm{C} 4$ and $\mathrm{C} 5$, respectively. Continuous elution test elicited the peak release of vancomycin from the fibrin sealant at 48 hours, with continued release until 2 weeks. However, conditioned vancomycin release decreased to half or more on day 2, however, the sustainable release was measured over the therapeutic dose $(10-20 \mu \mathrm{g} / \mathrm{mL})$ for 5 days and 4 days in assays of half and total exchange of PBS.

Conclusion : This study suggests that fibrin sealant can provide an efficient vehicle for antibiotic drug release in a wide range of neurosurgical procedures and the safe and effective therapeutic dose will be at the concentration embedded of $4.167 \mathrm{mg} / \mathrm{disc}$ or more of vancomycin.

Key Words : Drug delivery system · Fibrin tissue adhesive · Disk diffusion antimicrobial tests · High-performance liquid chromatography · Surgical wound infection.

- Received : July 17, $2019 \bullet$ Revised : August 19, $2019 \bullet$ Accepted : August 27, 2019

- Address for reprints : Moon-Jun Sohn

Department of Neurological Surgery, Neuroscience \& Radiosurgery Hybrid Research Center, Inje University Ilsan Paik Hospital, Inje University College of Medicine, 170 Juhwaro, Ilsanseo-gu, Goyang 10380, Korea

Tel : +82-31-910-7730, Fax : +82-31-915-0885, E-mail : mjsohn@paik.ac.kr, ORCID : https://orcid.org/0000-0002-1796-766X

This is an Open Access article distributed under the terms of the Creative Commons Attribution Non-Commercial License (http://creativecommons.org/licenses/by-nc/4.0) which permits unrestricted non-commercial use, distribution, and reproduction in any medium, provided the original work is properly cited. 


\section{INTRODUCTION}

Surgical site infection (SSI) is a critical aspect in the surgical field. SSI occurs in $1.9 \%$ of surgeries, and varies among populations, surgical procedures, and instrumentation ${ }^{3,10,12,13,15,24)}$. SSI results in a significant morbidity and financial burden due to longer hospital stay, readmission, and repeated procedures ${ }^{3,5,18,34)}$. Even though most surgeons do their best to prevent it, SSI is still a challenging issue. SSI is emphasized especially when the surgical procedures include instrumentation or graft application ${ }^{24,29)}$. In an attempt to decline infection rate, local application of antibiotics is performed to maintain sufficiently high drug concentrations for durable periods in the surgical wound space. A variety of methods for local release have been used to deliver antibiotics, including direct application of vancomycin powder, polymethylmethacrylate (PMMA), bone grafts, and natural polymers. As one of the natural polymers, fibrin sealant is a familiar term in the neurosurgical field because many surgeons use it for hemostasis, preventing cerebrospinal fluid (CSF) leakage, controlling sinus bleeding in cranial surgery, or sealing dead space in spinal surgery. Fibrin sealant consists of fibrin, thrombin, factor XIII, and Ca++. Since it is naturally present in the human body, it has several advantages over other sealants due to low inflammatory response, lack of immune interference, and natural absorption. Recent studies indicate that fibrin sealant acts as a biodegradable drug delivery agent as well as in hemostasis $^{2,25,28,31)}$. Morishima et al. ${ }^{23)}$ and Ozaki et al. ${ }^{25)}$ reported that vancomycin-impregnated fibrin sealant effectively prevented prosthetic graft infection. Tofuku et al. ${ }^{31)}$ reported that the zone of inhibition for antibiotic-impregnated fibrin sealant is maintained for at least 4 weeks. However, the ideal antibiotic release from antibiotic-impregnated polymers still needs to be investigated. Further, clinical evidence regarding drug delivery characteristics of fibrin sealant is still lacking. This study will support the use of fibrin sealant as a drug delivery agent. The outcome of this study will determine the optimal dosage in clinical use and the relationship between time and the antibiotic concentration in the fibrin sealant for the management of SSI.

\section{MATERIALS AND METHODS}

This study was approved by the Institutional Review Board of Inje University Ilsan Paik hospital (NON2018-005), which waived the need to obtain patient consent because this is in vitro study about antibiotic release from fibrin sealant. This study was designed to investigate bacterial susceptibility and the sustainability of vancomycin-impregnated fibrin sealant for two consecutive weeks. The fibrin sealant used in this study was obtained from Beriplast ${ }^{\circledR}$ kit (CSL Behring GmbH, Marburg, Germany). A disc diffusion test was performed using Blood Agar Plates (BAPs) and methicillin-resistant Staphylococcus aureus (MRSA) (ATCC29213) for the measurement of bacterial inhibition. Elution test was performed for measuring continuous drug release using high-performance liquid chromatography (Agilent 1200 Infinity LC, Agilent technologies, Santa Clara, CA, USA).

\section{Disc diffusion test}

Each set of vancomycin-impregnated fibrin sealants was prepared. Materials were subjected to agar diffusion testing to determine whether the sealant pieces possessed antimicrobial activity. The test materials also included drug-free mixtures without vancomycin as controls. These test materials were placed on agar plates and inoculated with a clinical biofilmforming strain of MRSA. The materials were incubated aerobically in an incubation chamber at $37^{\circ} \mathrm{C}$. Further, to determine the duration of antimicrobial activity, entire mixtures of vancomycin-impregnated fibrin sealant were incubated for 1 day. During the incubation period, the antibiotic diffused into the bacteria-seeded agar. The zone of bacterial growth inhibi-
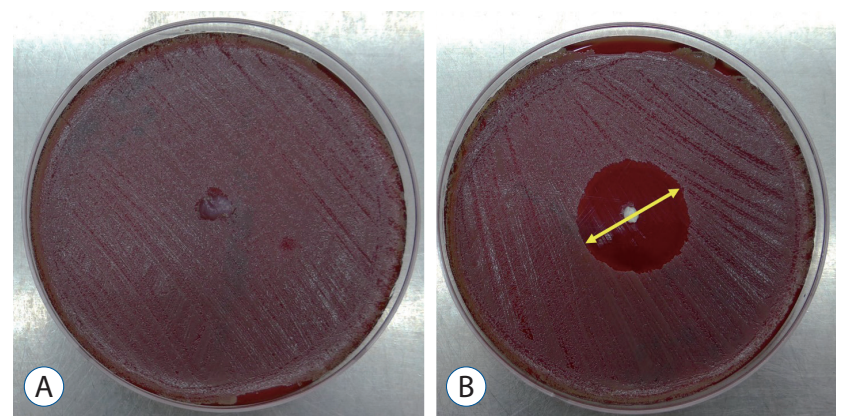

Fig. 1. Comparison of disk diffusion test inoculated with Staphylococcus aureus (MRSA, ATCC29213) on Blood Agar Plates for (A) control (fibrin sealant without vancomycin) on the left and (B) concentration 1 (C1, fibrin sealant with vancomycin) on the right. The diameter of the clear zone was measured after 24 hours (arrow). 
tion was evident as a clear region surrounding the test material using MRSA strains in this study (Fig. 1). The minimum inhibitory concentration (MIC) was defined as the lowest concentration of vancomycin that inhibited visible growth of the test bacteria.

\section{Organism preparation}

MRSA were used in this study. MRSA were preserved in skimmed-milk broth stored at below $-65^{\circ} \mathrm{C}$ until use. The culture was incubated in sterile BAPs at $37^{\circ} \mathrm{C}$ for 24 hours to obtain isolated colonies.

\section{Determination of the MIC of MRSA}

Using the VITEK AST-P601 (bioMérieux, Marcy l'Etoile, France) card and the VITEK Vitek-two, the MIC of vancomycin on MRSA was measured using the standard protocol. In these species, the MIC was $1.0 \mu \mathrm{g} / \mathrm{mL}$. In the susceptibility test for vancomycin, a clear zone diameter greater than $15 \mathrm{~mm}$ was determined as acceptable.

\section{Zone of inhibition test for antibacterial activity}

\section{Preparation of biodegradable drug delivery materials}

Fibrin sealant was removed from the refrigerator and allowed to thaw for at least 30 minutes before application for a better mixture. Two concentrations of vancomycin (Hanomycin, Samjin pharmaceuticals, Seoul, Korea) were used in this study : $500 \mathrm{mg}$ of vancomycin was dissolved in $1 \mathrm{~mL}$ and in $2 \mathrm{~mL}$ of sterile saline solution for concentrations (a) $500 \mathrm{mg} / \mathrm{mL}$ and (b) $250 \mathrm{mg} / \mathrm{mL}$ respectively. For each concentration, $1 \mathrm{~mL}$ of the vancomycin solution was added to the thrombin solution ( 2 $\mathrm{mL}$ ) of fibrin sealant kit and shaken to incorporate. The mixture was then prepared as per fibrin sealant kit recommendations with the fibrinogen solution $(3 \mathrm{~mL})$. The sealant (Thrombin-vancomycin mixture+fibrinogen) formed $6 \mathrm{~mL}$ in total that was then applied to cell culture wells to form uniformsized ( $0.1 \mathrm{~mL}, 6.4 \mathrm{~mm}$ diameter) discs. Each disc contained approximately $8.33 \mathrm{mg}$ (defined as concentration 1 [C1], n=9) and $4.167 \mathrm{mg}(\mathrm{C} 2, \mathrm{n}=9)$ of vancomycin for (a) and (b) respectively.

\section{Five different consecutive dose diluted antibiotic-loaded} fibrin sealant discs

In addition to the two different antibiotic-diluted discs, further consecutive diluted antibiotic-loaded fibrin sealant discs were used to compare the zone of inhibition among different concentrations of antibiotic-loaded discs. Standard procedure for the preparation of MRSA cell culture and inoculation onto BAP was performed as described previously for the zone of inhibition method. In total, $500 \mathrm{mg}$ of vancomycin was dissolved in $1 \mathrm{ml}$ of sterile saline solution. This was diluted by $10-$ fold (C3, n=3), 100-fold (C4, n=3), and 1000-fold (C5, n=3) to incorporate into fibrin sealant. The antibiotic-loaded fibrin sealant was then molded into $0.1 \mathrm{~mL}$ (6.7-7.0 $\mathrm{mm}$ in diameter) discs with concentrations of $0.83 \mathrm{mg} / \mathrm{disc}, 0.083 \mathrm{mg} / \mathrm{disc}$, and $0.0083 \mathrm{mg} / \mathrm{disc}$, for $\mathrm{C} 3, \mathrm{C} 4$, and $\mathrm{C} 5$ respectively. The discs were placed in the middle of MRSA inoculated BAPs in triplicate and were incubated at $37^{\circ} \mathrm{C}$ for 24 hours.

\section{Preparation of plates}

MRSA (ATCC25923) culture was diluted to a cell density of 0.5 to 0.6 Macf using Au sterile saline $(\mathrm{NaCl})$ solution as measured with VITEK Denscichek. The final bacterial suspension was inoculated onto sterile BAPs incubated at $37^{\circ} \mathrm{C}$ overnight. Antibiotic-loaded sealant discs $(100 \mu \mathrm{L})$ were placed at the center of the agar plates in triplicate. A set of triplicates with no treatment (no sealant, no vancomycin loaded sealant) was used as the control. The zone of inhibition, which diameter of clear circular zone, was measured at 24 hours after incubation time. During the experimental period, all plates were incubated at $37^{\circ} \mathrm{C}$. The area of the zone of inhibition was then calculated as described by Cashman et al. ${ }^{8)}$.

\section{Elution assays}

The in vitro drug release profile was studied with two different sets of drug concentrations $(8.33 \mathrm{mg} / \mathrm{disc}$ and 4.167 $\mathrm{mg} /$ disc, $\mathrm{C} 1$ and $\mathrm{C} 2$, respectively) and the data were subjected to kinetic interpretation. The drug release profiles of loaded and unloaded fibrin sealant were measured at different time schedules during 24 hours, 48 hours, 3 days, 5 days, 7 days, and 2 weeks. This quantitative test measured the sustained release and change in concentration over time using HPLC.

\section{Measurement of Cumulative vancomycin release during 2} consecutive weeks

Antibiotic impregnated fibrin sealant discs with two different concentrations of vancomycin were used; the $\mathrm{C} 1$ and $\mathrm{C} 2$ discs contained approximately $8.33 \mathrm{mg} /$ disc $(0.1 \mathrm{~mL})$ and 4.167 $\mathrm{mg} /$ disc of vancomycin, respectively. Discs were produced using the same method as in the disk diffusion test. To deter- 


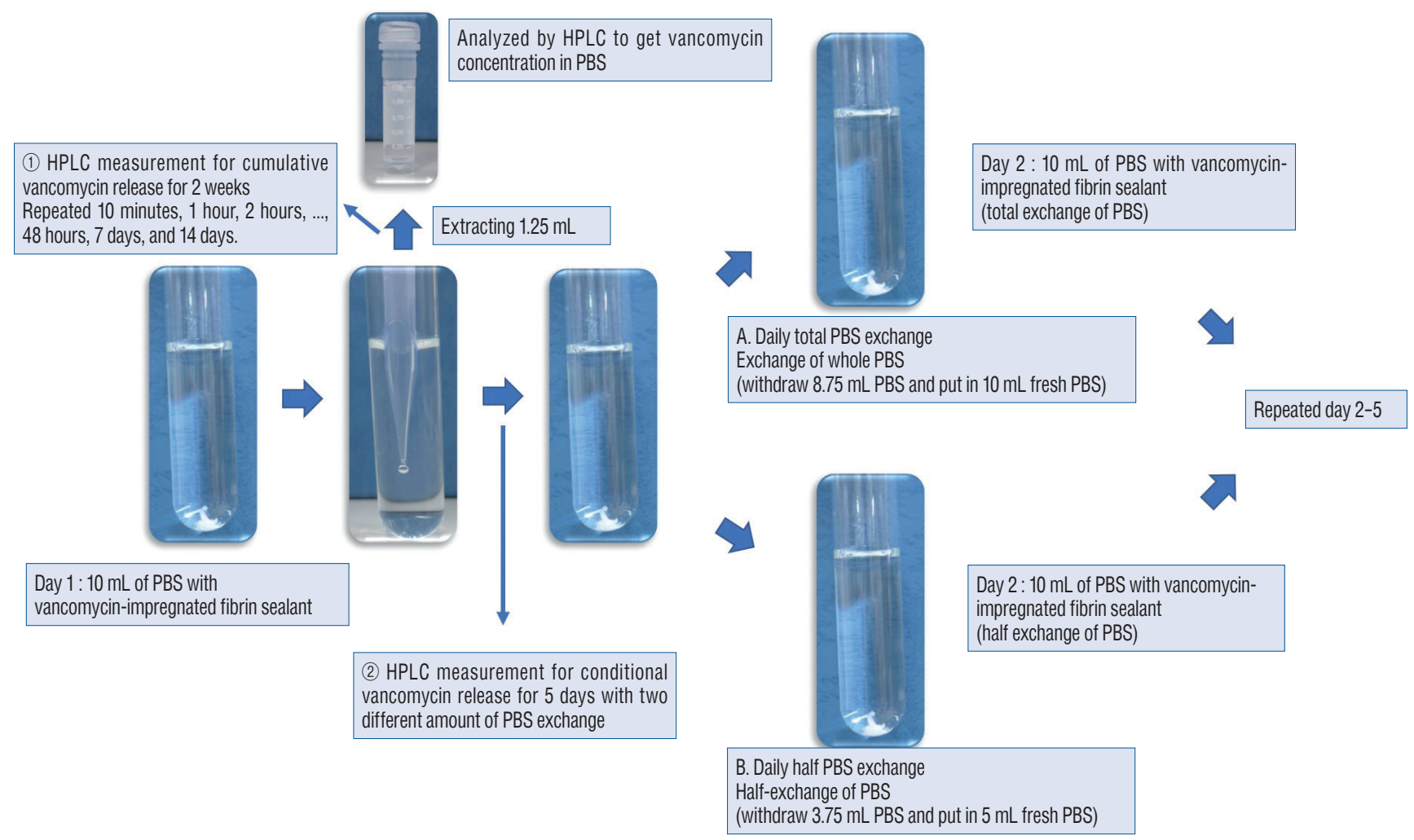

Fig. 2. HPLC measurement for (1) cumulative vancomycin release for 2 consecutive weeks and (2) two different Conditional vancomycin release each of daily total and half PBS exchange from day 1 to day 2 and sample collection for analysis. HPLC : high-performance liquid chromatography, PBS : phosphate buffer solution.

mine the release characteristics of vancomycin from the two different vancomycin concentrations loaded sealant discs (Cl and C2), each of the pre-prepared sealant discs (1 disc per tube) was placed in $10-\mathrm{mL}$ tubes containing $10 \mathrm{~mL}$ of phosphate buffer solution (PBS), in triplicate. The dissolution set was incubated at $37^{\circ} \mathrm{C}$ and at 0 minute, 10 minutes, 1 hour, 2 hours, 4 hours, 8 hours, 12 hours, 24 hours, 48 hours, 7 days, and 14 days, $500-\mu \mathrm{L}$ aliquots were collected. Vancomycin concentrations in the samples were measured using HPLC. The dissolution sets were then incubated at $37^{\circ} \mathrm{C}$.

The paired triple sets of tube with each $\mathrm{C} 1$ and $\mathrm{C} 2$ loaded fibrin sealant disc in $10 \mathrm{~mL}$ of PBS were used in order to measure the rate of continuous vancomycin release. Each of $1.25 \mathrm{~mL}$ PBS samples were taken from each set of tube at 10 minutes, 1 hour, 2 hours, 4 hours, 8 hours, 12 hours, 24 hours, 48 hours, 7 days, and 14 days (Fig. 2). After each sampling, $1.25 \mathrm{~mL}$ of PBS was replaced. To avoid a calculation error due to replacement, we corrected the PBS volume when we measured the released vancomycin. The equation of calibration curve for HPLC analysis was $y=15.797 x+90.264\left(R^{2}=1.000\right)$ (Fig. 3).
Elution assay for Conditional release of vancomycin with two different PBS turnovers (daily total versus daily half exchange of PBS)

Although being an in vitro study, we simulated in-vivo conditions by assuming body fluid exchange which might be caused by drain out, pooled hematoma, or serous fluid. Two types of conditional elution test were performed with a PBS exchanges in two different ways (Fig. 2). A : measurement of conditional vancomycin release with daily total PBS exchange test : to assess the release rate with total fluid turnover, the solution was replaced with $10 \mathrm{~mL}$ of fresh PBS at 24 hours intervals for 5 consecutive days. B : measurement of conditional vancomycin release with daily half PBS exchange : to assess the release rate with half fluid turnover, $5 \mathrm{~mL}$ of the solution was replaced with $5 \mathrm{~mL}$ of fresh PBS at 24 hours intervals for 5 consecutive days. The $1.25 \mathrm{~mL}$ sample was taken at the time stated above and stored at $-60^{\circ} \mathrm{C}$ for a maximum of three days before being assessed for HPLC analysis. 
Fibrin Sealant and Antibiotic Delivery I Shin DW, et al.

\begin{tabular}{ccccccc}
\hline Nominal conc. $(\mu \mathrm{g} / \mathrm{mL})$ & Area & Replicates & Measured conc. $(\mu \mathrm{g} / \mathrm{mL})$ & RSD $(\%)$ & Accuracy $(\%)$ \\
\hline 0 & 0 & & & & \\
20 & 344.9 & 6 & 16.1 & 1.0 & 80.6 \\
50 & 851.8 & 2 & 48.2 & 1.0 & 96.4 \\
100 & 1704.7 & 2 & 102.2 & 0.7 & 102.2 \\
\hline 200 & 3343.8 & 2 & 206.0 & 0.1 & 103.0 \\
\hline 500 & 7949.2 & 6 & 497.5 & 0.3 & 99.5 \\
\hline
\end{tabular}

Calibration curve : $y=15.797 x+90.264\left(R^{2}=1.000\right)$

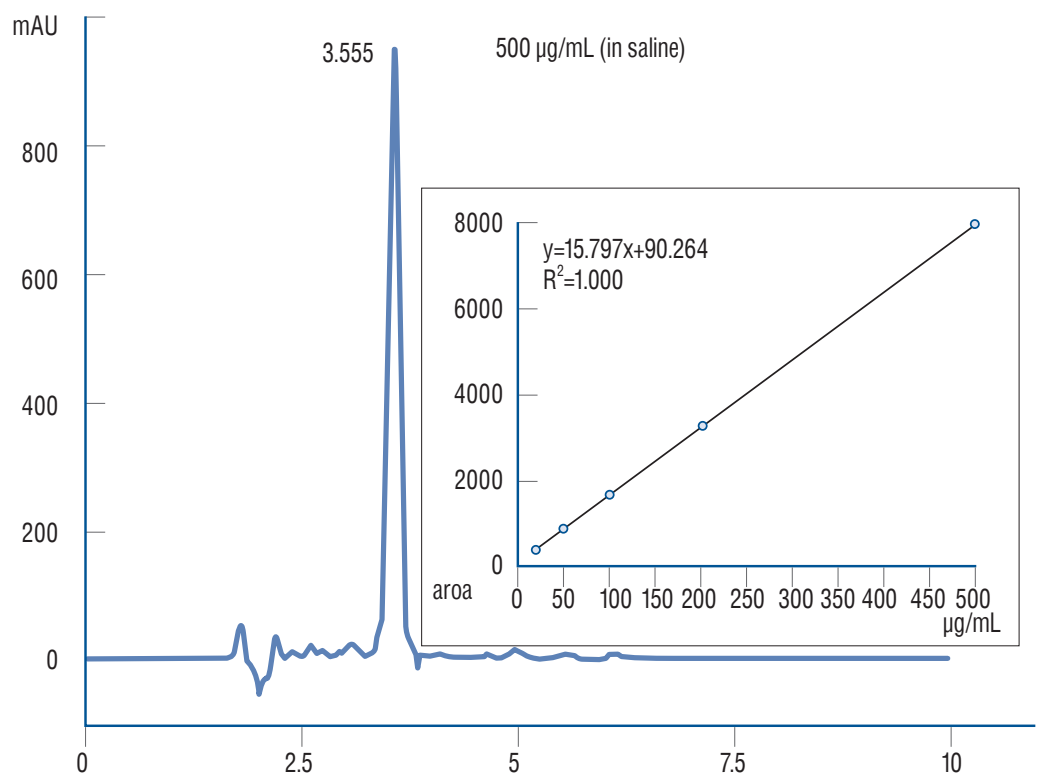

Fig. 3. Relationship between peak area and concentration of vancomycin release measured and the process of calibrating concertation of vancomycin release in each PBS sampling for HPLC analysis. RSD : relative standard deviation, PBS : phosphate buffer solution, HPLC : high-performance liquid chromatography.

\section{Statistical analysis}

The results are measured as mean and standard deviation. Both paired and unpaired t-test and one-way analysis of variance (ANOVA) were used as the analysis method. In all cases, a significance level of $p<0.05$ denoted statistical significance. All statistical analyses were performed using GraphPad Prism 8 (GraphPad Software, Inc., San Diego, CA, USA).

\section{RESULTS}

\section{Antibacterial measurement of zone of inhibition in disk-diffusion test}

The summary of measurement of zone inhibition in each sets of blood agar plate was shown in Table 1 . The average diameter of clear zone for antibacterial growth inhibition was $2.54 \pm 0.07 \mathrm{~cm}$ and $2.61 \pm 0.12 \mathrm{~cm}$ in BAP diffusion test with two fibrin sealant discs which loaded with higher dose of vancomycin concentrations $(8.33 \mathrm{mg} / \mathrm{disc}$ of $\mathrm{Cl}$ and $4.167 \mathrm{mg} / \mathrm{disc}$ of $\mathrm{C} 2$, respectively). For $\mathrm{C} 3, \mathrm{C} 4$, and $\mathrm{C} 5$, the zone of inhibition was correlated to the dilutions with gradual decline of mean diameter of $2.13 \pm 0.15 \mathrm{~cm}, 1.67 \pm 0.06 \mathrm{~cm}$, and $1.23 \pm 0.15 \mathrm{~cm}$, respectively (Table 1 ).

Under the $4.167 \mathrm{mg} / \mathrm{disc}$, the vancomycin concentration and zone of inhibition seemed to follow a dose-dependent relationship ( $p<0.05$, ANOVA). Furthermore, post-hoc analysis by Tukey's HSD showed a statistically significant different among the five groups except for C1-C2, and C4-C5 (Fig. 4). All concentrations except for C5 (0.0083 mg/disc) fulfilled the 
Table 1. Measurement in diameter $(\mathrm{cm})$ of zone of inhibition in disk diffusion test

\begin{tabular}{|c|c|c|c|c|c|}
\hline \multirow{2}{*}{ Set } & \multicolumn{5}{|c|}{ Types of disc concentration } \\
\hline & $\mathrm{C} 1(\mathrm{n}=9,8.33 \mathrm{mg} / \mathrm{mL})$ & $\mathrm{C} 2(\mathrm{n}=9,4.16 \mathrm{mg} / \mathrm{mL})$ & $\mathrm{C} 3(\mathrm{n}=3,0.83 \mathrm{mg} / \mathrm{mL})$ & $\mathrm{C} 4(\mathrm{n}=3,0.083 \mathrm{mg} / \mathrm{mL})$ & $\mathrm{C} 5(\mathrm{n}=3,0.0083 \mathrm{mg} / \mathrm{mL})$ \\
\hline 1 & $2.3 / 2.6 / 2.5$ & $2.3 / 2.8 / 2.4$ & 2.1 & 1.7 & 1.4 \\
\hline 2 & $2.2 / 2.6 / 2.9$ & $2.4 / 2.8 / 2.6$ & 2.3 & 1.6 & 1.2 \\
\hline 3 & $2.7 / 2.4 / 2.7$ & $2.8 / 2.7 / 2.7$ & 2.0 & 1.7 & 1.1 \\
\hline Mean & 2.54 & 2.61 & 2.13 & 1.67 & 1.23 \\
\hline SD & 0.07 & 0.12 & 0.15 & 0.06 & 0.15 \\
\hline
\end{tabular}

SD : standard deviation

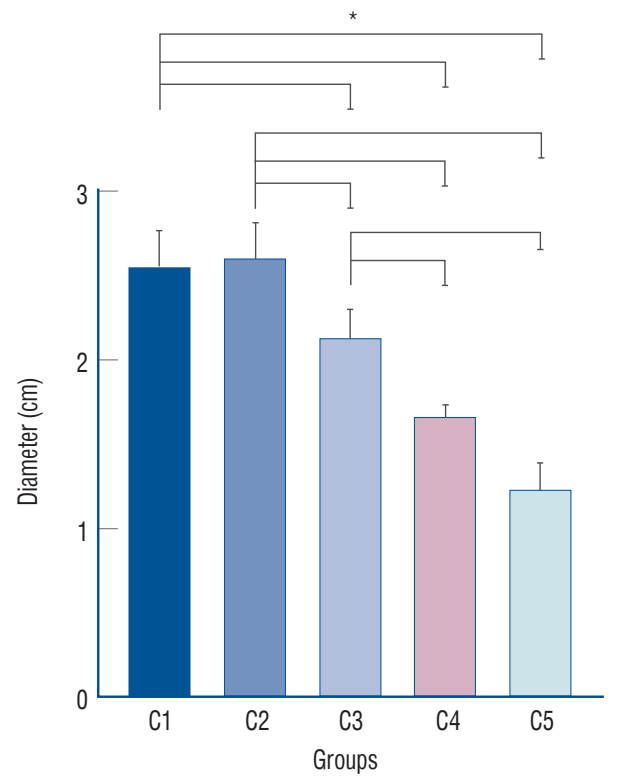

Fig. 4. Comparison in zone of inhibition test for five different concentrations with respective standard deviation. ${ }^{*} p<0.05$ in one way ANOVA, post-hoc analysis by Tukey's HSD showed $p<0.05$ except for $\mathrm{C} 1-\mathrm{C} 2$ and C4-C5. ANOVA : analysis of variance.

threshold of the antibiotic susceptibility test at a clear zone diameter of $15 \mathrm{~mm}$.

\section{Elution test for cumulative and conditional van- comycin-release measurement using HPLC}

Irrespective of release behavior, all results showed a sustained release of vancomycin from the vancomycin-loaded sealant. After 2 weeks of observation, the vancomycin impregnated fibrin sealant discs had still not degraded.

Measurement of cumulative antibiotic release (day 0 to 2 weeks)

The results of the cumulative measurement of vancomycin

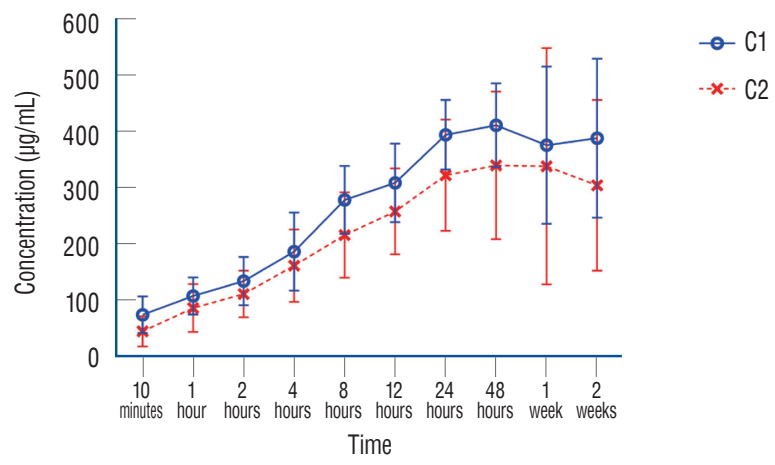

Fig. 5. Continuous release test - average vancomycin concentration with standard deviation $(\mu \mathrm{g} / \mathrm{mL})$ from vancomycin loaded fibrin sealant for $\mathrm{C1}$ and C2 over 2 weeks of study periods.

release lasting for 2 weeks are as follows. After inoculation of fibrin sealant discs containing two different concentrations of vancomycin ( $\mathrm{C} 1$ and $\mathrm{C} 2$ ) into each set of PBS tubes, vancomycin release in PBS lasted for 10 minutes and was measured more than $50 \mu \mathrm{g} / \mathrm{mL}$ in both sets of PBS. This cumulative concentration was comparably high over therapeutic drug monitoring (TDM) which therapeutic efficacy dose recommended $10-20 \mu \mathrm{g} / \mathrm{mL}$ in blood. The cumulative antibiotic release was continuously increased for 48 hours and reached maximum concentration at 48 hours. The average maximum concentration reached at $400 \mu \mathrm{g} / \mathrm{mL}$ and $340 \mu \mathrm{g} / \mathrm{mL}$ of Cl and C2 elution test, respectively. Then, the cumulative concentration was maintained constant for 2 consecutive weeks (Fig. 5).

Measurement of conditional vancomycin release with daily total PBS exchange (day 1 to day 5)

The result of the conditional vancomycin release with daily total PBS exchange for 5 days were as follows. The fibrin sealant discs containing two different concentrations of vancomycin (C1 and $\mathrm{C} 2)$ were inoculated into each set of PBS tubes. The 


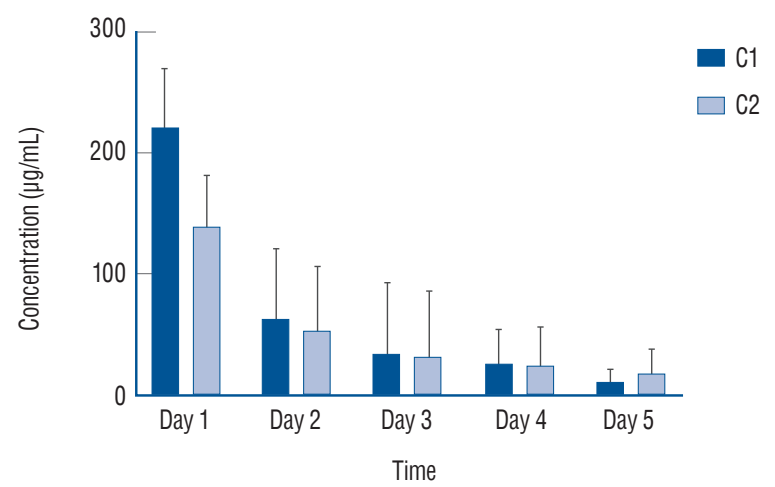

Fig. 6. Average vancomycin release $(\mu \mathrm{g} / \mathrm{mL})$ from vancomycin loaded fibrin sealant in $\mathrm{C} 1$ and $\mathrm{C} 2$ with total solution exchange every 24 hours for 5 days (e.g., rapid drainage by JP drain or significant bleeding in operation field).

vancomycin release in PBS on day 1 reached more than 200 $\mu \mathrm{g} / \mathrm{mL}$ of vancomycin in $\mathrm{PBS}$ tube of $\mathrm{C} 1$ whereas the other tube set of $\mathrm{C} 2$ released more than $100 \mu \mathrm{g} / \mathrm{mL}$. After total PBS exchange in each set of PBS tubes, the vancomycin release concentration on day 2 was decreased by almost half compared to the previous day. The mean concentrations of the antibiotic release in $\mathrm{C} 1$ and $\mathrm{C} 2$ were decreased to $26.12 \mu \mathrm{g} / \mathrm{mL}$ and 24.06 $\mu \mathrm{g} / \mathrm{mL}$, respectively, on the third and fourth days following continuous daily exchange. Until three consecutive total exchange of PBS, however, the vancomycin concentration in PBS was maintained more than that of TDM $(10-20 \mu \mathrm{g} / \mathrm{mL})$. On the last day 5 , the average concentration of vancomycin was dropped to less than $10 \mu \mathrm{g} / \mathrm{mL}$ (Fig. 6) following daily total PBS exchange.

Measurement of conditional vancomycin release with daily half PBS exchange (day 1 to day 5 )

The measurement results from daily half PBS exchange were as follows. On day 1 after inoculation of both $\mathrm{C} 1$ and $\mathrm{C} 2$ impregnated fibrin sealant discs in each set of PBS tubes, the vancomycin release was measured consistently about 200 $\mu \mathrm{g} / \mathrm{mL}$ of vancomycin. Similarly, the vancomycin release rate decreased to half on day 2 following the half exchange of PBS, but the conditional release rate remained at a concentration similar to day 2 until day 3. As following consecutive daily half exchange, the vancomycin concentration was gradually decreased to $20.34 \mu \mathrm{g} / \mathrm{mL}$ and $17.58 \mu \mathrm{g} / \mathrm{mL}$ on day 4 and day 5 , respectively and remained at a concentration higher than TDM in blood (Fig. 7).

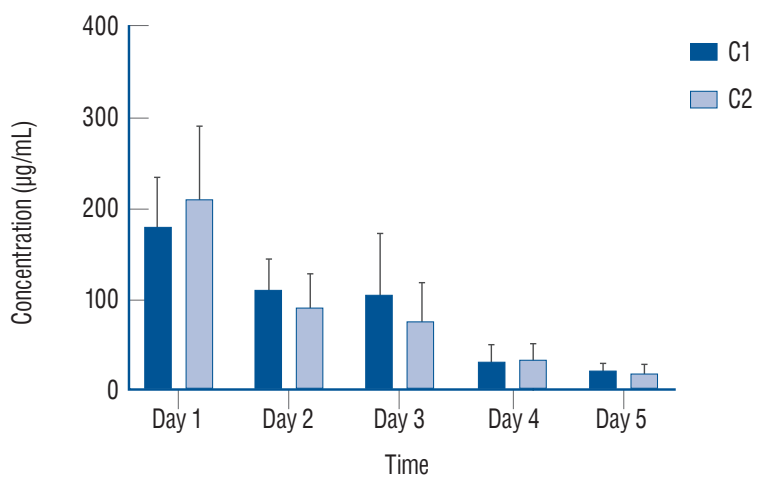

Fig. 7. Average vancomycin release $(\mu \mathrm{g} / \mathrm{mL})$ from vancomycin loaded fibrin sealant in $\mathrm{C} 1$ and $\mathrm{C} 2$ with half solution exchange every 24 hours for 5 days (e.g., natural drainage by JP drain or slowly body fluid exchange).

\section{Summary of results}

In summary, $\mathrm{C} 1(8.33 \mathrm{mg} / \mathrm{disc})$ and $\mathrm{C} 2(4.167 \mathrm{mg} / \mathrm{disc}) \mathrm{did}$ not show a difference in both the disk-diffusion test and elution assay. This suggests that the optimal vancomycin concentration for clinical application is likely at least more than 4.167 $\mathrm{mg} /$ disc for therapeutic purpose. According to the elution assay, released vancomycin concentration was higher than TDM even in 10 minutes after application. Further, the peak amount of released vancomycin was achieved in 24-48 hours. Additionally, in both daily total and half exchange of PBS, the vancomycin concentration was over the therapeutic concentration for 4 days and 5 days, respectively. Cumulative release of vancomycin will last more than 2 weeks and will maintain a sufficient therapeutic antibiotic level over MIC.

\section{DISCUSSION}

Microvascular networks in uncomplicated surgical wound enhanced to reach sufficient intravenous antibiotic level by angiogenesis in healing process. However, complicated wounds such as surgical dead space filled with body fluid collection prohibit to maintain intravenous antibiotic concentration. In this compromised surgical wound, intravenous administration of antibiotics during postoperative periods often limits the local drug concentrations above MIC and is also influenced by systemic toxicity in general (e.g., diarrhea, rash, or phlebitis, etc.), renal toxicity, and leukopenia in long term use due to lack of antibiotics penetration into the surgical wound $d^{7}$. 
Local antibiotics application has been studied as an alternative to solve this problem. On the other hand, local application of antibiotics could maintain the drug concentration for a longer period with less systemic toxicity ${ }^{1)}$. According to their specific method of use, topical antibiotic application can be divided into three group; 1) direct powder application like vancomycin, 2) use of non-absorbable products such as PMMA, and 3) biodegradable agents including autologous bone grafts, synthetic polymers, and natural polymers like fibrin sealant. In the neurosurgical field, for both cranial and spinal surgery, many clinicians are facing the prevention and treatment of postoperative surgical wound infection related with CSF leakage via dura suture, sinus air cells that often resulted from the reconstruction of trans-sphenoidal surgery, skull base surgery, brain and spine trauma, and implantation of device (e.g., shunt operation or spinal surgery). A biofilm is defined as a community of micro-organisms attached to a surface, and embedded within a matrix of extracellular polymeric substances. As instrumentation is a good source of biofilm formation, it is important that antibiotic concentrations be maintained above the MIC for several days after surgery, especially if the procedure involves instrumentation or vascular or artificial grafts ${ }^{626)}$. Traditionally, antibiotic impregnated PMMA has been widely used in complicated orthopedic surgery such hip and long bone surgical infection. This drug delivery carrier has been proved to use as a vehicle of locally sustainable drug release in surgical dead space. However, this non-biodegradable device has a disadvantage to be removed after the use despite of its advantage of stable local drug release ${ }^{17}$. Topical vancomycin powder application is simple and the easiest way to provide but has serious limitation of sustainable release due to lack of delivery carrier. Followings are summaries in cons and pros of the ways for topical antibiotic application and its use of delivery vehicles.

First, direct antibiotic powder application is one of the easiest methods to reduce postoperative infection in both cranial and spinal surgery ${ }^{3,11,12,26,29,30)}$. Direct powder application is valuable for cost saving by reducing repeat admission and extra surgeries ${ }^{11)}$. It can enhance the local concentration of vancomycin while blood vancomycin levels are still undetectable. Adverse effects of vancomycin are rarely reported. Khan et al. ${ }^{18)}$ reported that applying vancomycin powder in surgical field provides absolute risk reduction and a relative risk reduction of $2.8 \%$ and $68 \%$, respectively. In other words, the chance of SSI is 2.99 times higher in the control group ${ }^{18)}$. The limitation of vancomycin powder is a lack of sustainable carrying vehicle which means that the drug concentration cannot be maintained in case of rapid fluid exchange conditions on the operation bed (e.g., significant postoperative bleeding, negative pressure drainage of barovac, or body fluid exchange).

In terms of providing an effective use of local drug release, therefore, PMMA has been widely used as drug delivery materials, especially in orthopedic and vascular graft surgeries since 1970s. It can effectively fill the surgical dead space as well as release antibiotics; it can also be made in a variety of sizes or shapes so that surgeons can apply them according to different situations. Many studies have described antibiotic release from PMMA. An in vitro study by Charlton-Ouw et al. ${ }^{9)}$ reported the efficacy of PMMA when combined with various antibiotics that were frequently used in abdominal graft surgery. However, it could also be a source of infection or form a granulation tissue, provided it remains in the soft tissue; re-operation is essential to remove the beads ${ }^{4,14,21)}$.

Finally, in an attempt to solve the problems posed by PMMA and vancomycin powder application, surgeons have investigated biodegradable materials. In addition to bone graft and substances such as calcium sulfate, hydroxyapatite, demineralized bone matrix, as a biodegradable carriers synthetic and natural polymers are used including synthetic hydrogel, poly-lactic acid, poly-lactic-co-glycolic acid, and collagen sponge and fibrin sealant, etc. Fibrin sealant, one of the biodegradable polymers, has been employed in surgical drug delivery systems. The FDA approved its commercial use as a hemostat in $1998^{22}$. It has since been used as a topical hemostat for sealing the dura mater incision line, or filling dead space in spinal surgery ${ }^{2,16,23,25,28)}$. In case of venous sinus injury during craniotomy, many neurosurgeons use this item to achieve bleeding control as well as to physically defend the sinuses ${ }^{27,32)}$. Furthermore, when the frontal sinus is opened, antibiotic-impregnated fibrin glue replaces gentamycin soaked gel foams, thus packing the sinus ${ }^{33)}$. There have been several reports about the clinical use of fibrin sealant as a drug delivery agent; however, previous studies have usually focused on the zone of inhibition test to show the effectiveness of fibrin glue with antibiotics ${ }^{8)}$.

In this study, we propose the optimal therapeutic range of vancomycin concentration that impregnates fibrin sealant carriers to maintain over MIC or therapeutic dose and provides effective antibacterial effects in disk diffusion test and 
elution test. In general usage, practical concentration of $\mathrm{C} 1$ (8.36 mg/disc) and C2 (4.167 mg/disc) showed no statistical difference in antibacterial activity (zone of inhibition) and cumulative and conditioned release of therapeutic dose range for vancomycin in the results of the disk diffusion test and elution assay. The antibacterial inhibition zone from C1, C2 to C3 $(8.33,4.167$ and $0.83 \mathrm{mg} /$ disc, respectively) was measured from 2.54 to $2.13 \mathrm{~cm}$ but significantly declined at C4 and C5 (0.083 and $0.0083 \mathrm{mg} / \mathrm{disc}$ ) from 1.67 to $1.23 \mathrm{~cm}$ in its diameter. This indicates preclinical cutoff value (over $4.167 \mathrm{mg} / \mathrm{disc}$ ) of a dose concentration effective for the therapeutic purpose to maintain above the minimal inhibitory concentration. Comparably higher concentration of vancomycin-loading is more promising to maintain sustainable release in conditional setting of daily total PBS exchange over daily half exchanging. In cumulative measurement of HPLC assay, the vancomycin concentration was maintained sufficiently high enough to therapeutic range for two consecutive weeks. This natural polymer can maintain its continuous antibacterial activity until it is degraded over time for several weeks. However, it can be influenced by structural limitations of fibrin glue or a saturated state of affinity to vancomycin ${ }^{2}$.

An animal study by Kroez et al. ${ }^{19)}$ indicated that the antibody titer for fibrin and thrombin peaked at 2 weeks and then gradually decreased in rabbit liver. This was in agreement with the present study results. Fibrin sealant was degraded and replaced by granulation tissue within 9 weeks ${ }^{19)}$. To achieve complete eradication of infection, sustainable release of antibiotics to the dead space of the surgical wound is essential. Additionally, the antibiotic concentration should be maintained above the MIC for as long as possible to control infection efficiently. Our study revealed that even total exchange of PBS showed maintenance of vancomycin concentration over the MIC for at least four days. This suggests that if the body fluid is refreshed entirely (e.g., JP drainage or significant bleeding at the operation site), the fibrin glue can still release vancomycin for at least 4 days above the therapeutic dose. This indicates that the fibrin sealant can endure rapid turnover of body fluid. Total exchange and half exchange of PBS were performed assuming that surgical dead space usually changes its fluid component as serous fluid from surrounding tissue or vessels accumulates while postoperative drainage (e.g., baro-vac) removes fluid from the dead space. Our results emphasize that fibrin glue may maintain high concentrations of vancomycin in the surgical dead space in vivo even under total or half fluid exchange conditions. Moreover, if used in the dead space of surgical wounds, fibrin glue can also provide hemostasis and dead space filling along with antibiotic release.

As shown in this study, fibrin sealant can effectively release vancomycin in vitro environment at least 4 or 5 days at daily total and half exchange of PBS. These results can be affected by turnover rate of surgical space fluid. In our cumulative release measurement, fibrin sealant as a drug delivery vehicle can provide sustainable vancomycin release as high as $400 \mu \mathrm{g} / \mathrm{mL}$ at a maximum dose in 48 hours and maintained 2 weeks. $\mathrm{Cu}-$ mulative release of vancomycin will last more than 2 weeks and will maintain a sufficient therapeutic antibiotic level over MIC. However, conditional antibiotic release from fibrin sealant is diluted and reduced gradually for several days depending on PBS exchange rate mimicking the physiologic conditions such as drainage of postoperative bleeding or fluid through the surgical hemo-vac. This result implies that if the fluid turnover is slower than our conditional antibiotic elution tests, antibiotic embedded fibrin sealant can release antibiotics over therapeutic drug concentration as long as 2 weeks or more depending on the PBS exchange rate, which mimics physiological conditions such as postoperative bleeding or body fluid release. In this study, conditional elution test assumed that active bleeding or body fluid drainage occurred in surgical wound. Even under extreme in vitro conditions such as total exchange of PBS, effective concentration of vancomycin release over TDM remained for consecutive 4 days. As the body fluid drainage and its duration in vivo environment, the cumulative release of vancomycin can maintain a sufficiently higher level of therapeutic concentration than MIC for more than 2 weeks.

This Study was limited to 2 weeks of experiment and did not assume to obtain the result of the overall continuous antibiotic release during the period of fibrin glue degradation. Further investigation is needed to determine the trend of antibiotic release over a few weeks. Additionally, future studies should examine a variety of vancomycin concentrations to obtain the best concentration with fibrin sealant for outstanding clinical results. Moreover, this study focused only on vancomycin and not on other antibiotics. Other antibiotics with different chemical properties might show different pharmacokinetics or sustainability when combined with fibrin sealant as indicated by a previous study with $\mathrm{PMMA}^{20)}$. The relation- 
ship between time and concentration differs among antibiotics because of their hydrophobic/hydrophilic status or molecular weight ${ }^{2}$. Thus, further studies should include other widely used antibiotics such as gentamycin or cephalosporin. The last limitation is that the in-vitro model cannot exactly match and explain the reality of in-vivo situations. In addition, the drug-impregnated fibrin sealant is usually not sufficient to cover the dead space of a wide surgical wound. However, this study emphasizes the use of fibrin sealant as an effective drug delivery vehicle for topical application in neurosurgical procedures to prevent or control focal infection site when combined with antibiotics.

\section{CONCLUSION}

This in vitro study demonstrated the biodegradable fibrin sealant as a vehicle for sustainable antibiotic release in the test period. When simulating the in-vitro model of half and total fluid accumulation and resorption, fibrin glue could release vancomycin over the MIC for at least 4 or 5 days. This result emphasizes that fibrin glue is an effective drug delivery agent in addition to its benefits regarding hemostasis, sealing defects, and adhesive function, which have been well documented in many previous studies. Based on these experimental data, further investigations are required in vivo and in pilot clinical application to establish the optimal concentration of antibiotic load in the fibrin sealant. Further studies should also include a variety of medications such as gram-negative bacteria-covering antibiotics, antitumor agents, proteins, or antigens. Then, the fibrin sealant could be used as a drug delivery vehicle for neurosurgery as well as in other surgical fields.

\section{CONFLICTS OF INTEREST}

No potential conflict of interest relevant to this article was reported.

\section{INFORMED CONSENT}

This type of study does not require informed consent.

\section{AUTHOR CONTRIBUTIONS}

\author{
Conceptualization : MJS, CRC \\ Data curation : DWS, MJS \\ Formal analysis : DWS, MJS, CRC \\ Funding acquisition : MJS \\ Methodology : MJS, CRC \\ Project administration : MJS, CRC \\ Visualization : MJS, CRC \\ Writing - original draft : DWS, MJS \\ Writing - review \& editing : DWS, MJS, CRC, HWK, SWY
}

\section{- Acknowledgements}

This work was supported by a research grant from HANDOK Inc. (HANDOK-2014-156) and Inje industry Academy cooperation foundation. Very special thanks to Tae-Hyun Park, M.S., and Eun Young Kim for contributing to this paper.

\section{References}

1. Abdullah KG, Attiah MA, Olsen AS, Richardson A, Lucas $T H$ : Reducing surgical site infections following craniotomy: examination of the use of topical vancomycin. J Neurosurg 123 : 1600-1604, 2015

2. Ahmad E, Fatima MT, Hoque $M$, Owais $M$, Saleemuddin $M$ : Fibrin matrices: the versatile therapeutic delivery systems. Int J Biol Macromol 81 : 121-136, 2015

3. Anderson PA, Savage JW, Vaccaro AR, Radcliff K, Arnold PM, Lawrence $B D$, et al. : Prevention of surgical site infection in spine surgery. Neurosurgery 80(3S) : S114-S123, 2017

4. Aziz KT, Best MJ, Naseer Z, Skolasky RL, Ponnusamy KE, Sterling RS, et al. : the association of delirium with perioperative complications in primary elective total hip arthroplasty. Clin Orthop Surg 10 : 286-291, 2018

5. Bernatz JT, Anderson PA : Thirty-day readmission rates in spine surgery: systematic review and meta-analysis. Neurosurg Focus 39 : E7, 2015

6. Bhattacharya M, Wozniak DJ, Stoodley P, Hall-Stoodley L : Prevention and treatment of Staphylococcus aureus biofilms. Expert Rev Anti Infect Ther 13 : 1499-1516, 2015

7. Bowler $P G$, Duerden BI, Armstrong DG : Wound microbiology and associated approaches to wound management. Clin Microbiol Rev 14 : 244-269, 2001

8. Cashman JD, Jackson JK, Mugabe C, Gilchrist S, Ball K, Tredwell S, et al. : The use of tissue sealants to deliver antibiotics to an orthopaedic surgical site with a titanium implant. J Orthop Sci 18 : 165-174, 2013

9. Charlton-Ouw KM, Kubrusly F, Sandhu HK, Swick MC, Leake SS, Gulbis $\mathrm{BE}$, et al. : In vitro efficacy of antibiotic beads in treating abdominal vas- 
cular graft infections. J Vasc Surg 62 : 1048-1053, 2015

10. Dessy AM, Yuk FJ, Maniya AY, Connolly JG, Nathanson JT, Rasouli JJ, et al. : Reduced surgical site infection rates following spine surgery using an enhanced prophylaxis protocol. Cureus 9 : e1139, 2017

11. Emohare O, Ledonio CG, Hill BW, Davis RA, Polly DW Jr, Kang MM : Cost savings analysis of intrawound vancomycin powder in posterior spinal surgery. Spine J $14:$ 2710-2715, 2014

12. Eren B, Karagöz Güzey F, Kitis S, Özkan N, Korkut C : The effectiveness of pedicle screw immersion in vancomycin and ceftriaxone solution for the prevention of postoperative spinal infection: a prospective comparative study. Acta Orthop Traumatol Turc 52 : 289-293, 2018

13. Grabel ZJ, Boden A, Segal DN, Boden S, Milby AH, Heller JG : The impact of prophylactic intraoperative vancomycin powder on microbial profile, antibiotic regimen, length of stay, and reoperation rate in elective spine surgery. Spine J $19: 261-266,2018$

14. Hake ME, Young $H$, Hak DJ, Stahel PF, Hammerberg EM, Mauffrey C : Local antibiotic therapy strategies in orthopaedic trauma: practical tips and tricks and review of the literature. Injury 46 : 1447-1456, 2015

15. Hill BW, Emohare O, Song B, Davis R, Kang MM : The use of vancomycin powder reduces surgical reoperation in posterior instrumented and noninstrumented spinal surgery. Acta Neurochir (Wien) 156 : 749-754, 2014

16. Horowitz B, Busch $\mathrm{M}$ : Estimating the pathogen safety of manufactured human plasma products: application to fibrin sealants and to thrombin. Transfusion 48 : 1739-1753, 2008

17. Jennings JA, Carpenter DP, Troxel KS, Beenken KE, Smeltzer MS, Courtney HS, et al. : Novel antibiotic-loaded point-of-care implant coating inhibits biofilm. Clin Orthop Relat Res 473 : 2270-2282, 2015

18. Khan NR, Thompson CJ, DeCuypere M, Angotti JM, Kalobwe E, Muhlbauer MS, et al. : A meta-analysis of spinal surgical site infection and vancomycin powder. J Neurosurg Spine 21 : 974-983, 2014

19. Kroez $M$, Lang $W$, Dickneite $G$ : Wound healing and degradation of the fibrin sealant Beriplast $P$ following partial liver resection in rabbits.

Wound Repair Regen 13 : 318-323, 2005

20. Kummer A, Tafin UF, Borens 0 : Effect of sonication on the elution of antibiotics from polymethyl methacrylate (PMMA). J Bone Jt Infect 2 : 208-212, 2017

21. McConoughey SJ, Howlin RP, Wiseman J, Stoodley P, Calhoun JH : Comparing PMMA and calcium sulfate as carriers for the local delivery of antibiotics to infected surgical sites. J Biomed Mater Res B Appl Biomater $103: 870-877,2015$

22. Mintz PD, Mayers L, Avery N, Flanagan HL, Burks SG, Spotnitz WD :
Fibrin sealant: clinical use and the development of the University of Virginia Tissue Adhesive Center. Ann Clin Lab Sci 31 : 108-118, 2001

23. Morishima M, Marui A, Yanagi S, Nomura T, Nakajima N, Hyon SH, et al. : Sustained release of vancomycin from a new biodegradable glue to prevent methicillin-resistant Staphylococcus aureus graft infection. Interact Cardiovasc Thorac Surg 11 : 52-55, 2010

24. Najjar PA, Smink DS : Prophylactic antibiotics and prevention of surgical site infections. Surg Clin North Am 95 : 269-283, 2015

25. Ozaki S, Saito A, Nakaminami H, Ono M, Noguchi N, Motomura N : Comprehensive evaluation of fibrin glue as a local drug-delivery systemefficacy and safety of sustained release of vancomycin by fibrin glue against local methicillin-resistant Staphylococcus aureus infection. J Artif Organs $17:$ 42-49, 2014

26. Percival SL : Importance of biofilm formation in surgical infection. $\mathbf{B r} \mathbf{J}$ Surg 104 : e85-e94, 2017

27. Sekhar LN, Natarajan SK, Manning T, Bhagawati $D$ : The use of fibrin glue to stop venous bleeding in the epidural space, vertebral venous plexus, and anterior cavernous sinus: technical note. Neurosurgery 61(3 Suppl) : E51; discussion E51, 2007

28. Spotnitz WD : Fibrin sealant: past, present, and future: a brief review. World J Surg 34 : 632-634, 2010

29. Strom RG, Pacione D, Kalhorn SP, Frempong-Boadu AK : Decreased risk of wound infection after posterior cervical fusion with routine local application of vancomycin powder. Spine (Phila Pa 1976) 38 : 991-994, 2013

30. Theologis AA, Demirkiran G, Callahan M, Pekmezci M, Ames C, Deviren $V$ : Local intrawound vancomycin powder decreases the risk of surgical site infections in complex adult deformity reconstruction: a cost analysis. Spine (Phila Pa 1976) 39 : 1875-1880, 2014

31. Tofuku K, Koga H, Yanase M, Komiya S : The use of antibiotic-impregnated fibrin sealant for the prevention of surgical site infection associated with spinal instrumentation. Eur Spine J 21 : 2027-2033, 2012

32. Toyooka T, Otani N, Wada K, Tomiyama A, Ueno H, Fujii K, et al. : Effect of fibrin glue injection into the cavernous sinus for hemostasis during transcavernous surgery on the cerebral venous draining system. Oper Neurosurg (Hagerstown) 13 : 224-231, 2017

33. Yang L, Wu J, Zhang G, Zhao Z, Fan Z, Wang B : A novel technology: percutaneous injection of fibrin glue as a treatment for frontal sinus cerebrospinal fluid rhinorrhea. J Craniofac Surg 24 : 1646-1649, 2013

34. Yeramaneni S, Robinson C, Hostin R : Impact of spine surgery complications on costs associated with management of adult spinal deformity. Curr Rev Musculoskelet Med 9 : 327-332, 2016 